\title{
Nonlinear error scalar matrix improved affine projection sign algorithm
}

\author{
Weifeng Zhao ${ }^{1}$, Yun Huang ${ }^{1}$, Wensan Zheng ${ }^{2}$ \\ ${ }^{1}$ Asset Management Department, Chongqing University of Posts and Telecommunications, \\ Chongqing, 400065, China \\ ${ }^{2}$ School of communication and information engineering, Chongqing University of Posts and \\ Telecommunications, Chongqing, 400065, China
}

Keywords: Affine projection sign algorithm; robust adaptive algorithm; errror scalar matrix.

\begin{abstract}
Proposed is an affine projection sign algorithm with a nonlinear error scalar matrix to improve the robustness and the tracking performance against non-Gaussian impulsive interferences. The error scalar matrix scalars down the errors of some projection directions in the presence of impulsive noise. The major contribution of the letter is that variable error nonlinearity methods used in normalized least mean square (NLMS) can be applied to the scalar matrix with a little modification. An ideal scalar matrix is presented in the simulation environment of the two component Gaussian mixture noise model. Although a closed-form solution of the ideal matrix cannot be obtained in practice, it provides us a heuristic consideration about how to design the scalar matrix and theoretically best learning curves that the proposed method can achieve. We also discuss a practical method to approximate the optimal learning curve. Improved performance of the proposed algorithm is demonstrated in a system identification scenario.
\end{abstract}

\section{Introduction}

The family of normalized sign algorithms (NSAs) are known for their better robustness against nonGaussian impulsive noise in comparison with the conventional NLMS [1]. Their convergence performance can be further improved by using the nonlinear transformation of the error signal [2-4]: the switched norm algorithm in the applications of system identification and acoustic echo cancellation [2], the logarithmic transformation in the context of active noise control [5-7], and the tan transformation tested in system identification [8] etc. Unfortunately their major drawbacks are performance degradation with correlated input signals. It is known that the affine projection sign algorithm (APSA) achieves faster convergence performance over NSA against correlated input signal, lower computational complexity and better robustness than the conventional affine projection algorithm (APA) [9-10]. However, it shows poor steady-state performance. In this letter, we report a novel APSA with a nonlinear error scalar matrix, which is obtained by using the nonlinear transformation of error signals. Small scalar factors are given to error signals corrupted by impulsive noise, which eliminates the possibility of updating weight vector based on the wrong sign information of the error signals owing to the impulsive noise. Both ideal and practical scalar matrices are discussed and computational complexities are also derived. The performances of proposed algorithms are tested in a system identification scenario.

\section{Error scalar matrix APSA (ESM-APSA)}

Consider the desired signal $\mathbf{d}_{i}$ that arise from the system identification model $d_{i}=\mathbf{u}_{i}^{T} \mathbf{w}_{o}+v_{i}$, where $\mathbf{w}_{o}$ is an unknown system be estimated, $\mathbf{u}_{i}$ denotes the input vectors $\mathbf{u}_{i}=\left(u_{i}, u_{i-1}, \cdots, u_{i-L+1}\right)^{T}$, and $v_{i}$ is background noise plus impulsive noise. The a priori and a posteriori error vectors are $\mathbf{e}_{i}=\mathbf{d}_{i}-\mathbf{U}_{i}^{T} \mathbf{w}_{i-1}$ and $\mathbf{e}_{p, i}=\mathbf{d}_{i}-\mathbf{U}_{i}^{T} \mathbf{w}_{i}$, where the desired output signal $\mathbf{d}_{i}=\left(d_{i}, d_{i-1}, \cdots, d_{i-K+1}\right)^{T}$, the data matrix $\mathbf{U}_{i}=\left[\mathbf{u}_{i}, \mathbf{u}_{i-1}, \cdots, \mathbf{u}_{i-K+1}\right]$, and $\mathbf{w}_{i}$ is the estimate of $\mathbf{w}_{o}$ at iteration $i$. The optimization criterion with an error scalar matrix for APSA can be written as [9]

$$
\min _{\mathbf{w}_{i}}\left\|\mathbf{I}_{K} \mathbf{e}_{p, i}\right\|_{1} \quad \text { Subject to } \quad\left\|\mathbf{w}_{i}-\mathbf{w}_{i-1}\right\|_{2}^{2} \leq \mu^{2}
$$


Where $\mathbf{I}_{K}$ is the identity matrix, $\mu^{2}$ is the minimum disturbance constraint. Although it improves the convergence rate of the NSA by using past input vectors especially for correlated input signals [9], the same weights to past error signals are unbeneficial to the weight vector update. What we would like, in fact, is to make the error signals corrupted by impulsive noise contribute little or even none to the weight vector update. Therefore, we propose to use a scalar matrix $\lambda_{i}$ to replace the fixed matrix $\mathbf{I}_{K}$ in (1), where $\lambda_{i}=\operatorname{diag}\left\{\lambda_{i}, \lambda_{i-1}, \cdots, \lambda_{i-K+1}\right\}$ is a diagonal matrix, and its diagonal elements satisfy the following condition $0 \leq \lambda_{i-j+1} \leq 1,1 \leq j \leq K$. Using the method of Lagrange multipliers, the cost function can be obtained:

$$
J\left(\mathbf{w}_{i}\right)=\left\|\lambda_{i} \mathbf{e}_{p, i}\right\|_{1}+\gamma\left(\left\|\mathbf{w}_{i}-\mathbf{w}_{i-1}\right\|_{2}^{2}-\mu^{2}\right)
$$

Where $\gamma$ is the Lagrange multiplier, and $\|\cdot\|_{1}$ is 1 -norm of a vector. By setting the derivative of the cost function (2) with respect to the weight vector $\mathbf{w}_{i}$ to zero, and using the constraint in (1), we obtain the update equation:

$$
\mathbf{w}_{i}=\mathbf{w}_{i-1}+\frac{\mu \mathbf{U}_{i} \lambda_{i} \operatorname{sgn}\left(\mathbf{e}_{i}\right)}{\sqrt{\operatorname{sgn}\left(\mathbf{e}_{i}^{T}\right) \lambda_{i}^{T} \mathbf{U}_{i}^{T} \mathbf{U}_{i} \lambda_{i} \operatorname{sgn}\left(\mathbf{e}_{i}\right)}}
$$

Noted that the posterior error vector $\mathbf{e}_{p, i}$ is inaccessible in practice, it is reasonable to replace it by the a priori error vector $\mathbf{e}_{i}$ [9]. Equation (3) reduces to the conventional APSA when $\lambda_{i}$ is the identity matrix. Therefore it can be view as a generalization of APSA.

\section{Optimal nonlinear error scalar matrix (ONESM)}

In order to obtain the optimal scalar matrix, the simulation model of the impulsive noise should be introduced first. The interference $v_{i}$ can be modelled as a two component Gaussian mixture [3][7-10]: $v_{i}=n_{i}+k_{i} A_{i}$ where $n_{i}$ and $A_{i}$ are each zero-mean independent white Gaussian sequences with variances $\sigma_{n}^{2}$ and $\sigma_{A}^{2}$; $k_{i}$ is a Bernoulli random process, with a probability of success $P\left[k_{i}=1\right]=p_{r}$. The ideal nonlinear error scalar matrix can be obtained based on above model:

$$
\lambda_{i-j+1}=\left\{\begin{array}{ll}
1 & k_{i-j+1}=0 \\
0 & k_{i-j+1}=1
\end{array} \quad j=1,2, \ldots, K\right.
$$

According to (3) and (4), the error signals contaminated by impulsive noise are instantly scaled to zeros to eliminate their influences to the weight vector update. Although (4) can be simulated in computer simulations, it cannot be put into practice. That is because the two component Gaussian mixture model no longer holds in practice and the occurrence probability of the outliers is unavailable. But it can be viewed as the optimal learning curve reference in simulations. Because the optimality and the nonlinear transformation (4), this method can be termed as ONESM-APSA. It requires $K L+2 L+1$ multiplications and averaging $K L(2-p r)+L$ additions at each time instant. The computational complexity of APSA and its fast implementation method can be found in [11-12].

\section{Practical nonlinear error scalar matrix APSA (NESM-APSA)}

The major advantage of the proposed scheme is that various nonlinear error transformation methods in NLMS [2-7] can be easily modified and then applied to (3). We specifically discuss a low computational complexity method inspired by a nonlinear transformation used in [4]. The error scalar matrix can be written as:

$$
\lambda_{i-j+1}=1 /\left(1+\gamma\left|e_{i-j+1} /\left\|\mathbf{u}_{i-j+1} \mid\right\|^{2}\right), \quad j=1,2, \ldots, K\right.
$$

Where $e_{i-j+1}$ is the $j$ th element of the a priori error vector? As can be seen from (5), when the magnitude of the normalized error is small, the scalar factor $\lambda_{i-j+1} \approx 1$. On the other hand, a larger magnitude of the normalized error leads to the expected result $\lambda_{i-j+1} \approx 0 . \gamma$ Is a positive constant to balance the steepness and the gentleness degrees of the transformation: a large $\gamma$ results in a steep slope, and vice versa? Therefore, (5) act as an approximation of the ideal error scalar matrix (4). 
This algorithm requires $3 K L+4 K+2 L+1$ multiplications and averaging $2 K L+K+L$ additions at each time instant.

\section{Simulation results}

The performance of proposed algorithms was tested in a system identification scenario. The system to be identified is randomly generated with 512 taps, and the adaptive filter is assumed to have the same number of taps. The input signals were obtained by filtering a white, zero-mean, Gaussian random signal through a first-order AR system $G(z)=1 /\left(1-0.7 z^{-1}\right)$. The output signal-to-noise ratio (SNR) was set to $30 \mathrm{~dB}$ for additive Gaussian white noise. The probability of the occurrence of impulsive noise $p_{r}$ was chosen as 0.1 . We set the power of $A_{i}$ to $\sigma_{A}^{2}=1000 \sigma_{y}^{2}$ where $\sigma_{y}^{2}$ be the power of the system output $y_{i}=\mathbf{u}_{i}^{T} \mathbf{w}_{o}$. The projection order $K$ was set to 10 . The convergence performance was evaluated by the normalized MSD (NMSD NMSD $=10 \log _{10}\left(\left\|\tilde{\mathbf{w}}_{i}\right\|_{2}^{2} /\left\|\mathbf{w}_{o}\right\|_{2}^{2}\right.$ ) where $\tilde{\mathbf{w}}_{i}=\mathbf{w}_{i}-\mathbf{w}_{o}$. We compared proposed algorithms with NSA, APSA, and Scalar NLMS (SNLMS) in [7].The learning curves were obtained by ensemble averaging over 30 independent trails.

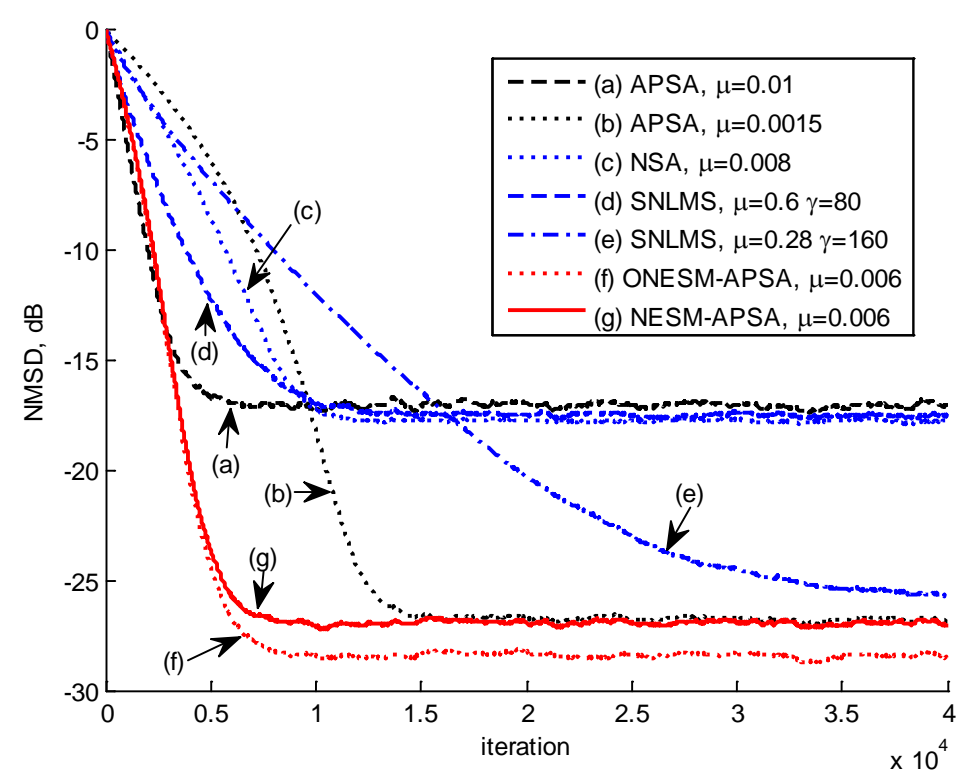

Fig. 1 NMSD learning curves for NSA, APSA, SNLMS, ONESM-APSA, and NESM-APSA $(\gamma=40)$

In the simulations, the values of step-size and $\gamma$ are chosen to have the same convergence rate or the same steady-state mismatch as the proposed scheme. As it can be seen from Fig. 1, by using nonlinear error transformation method, the SNLMS obtains a good initial convergence while the NSA converges slowly. However, the APSA achieves the fastest convergence using past input vectors. The ONESM-APSA and NESM-APSA show the same convergence with APSA but with a $10 \mathrm{~dB}$ lower steady-state error by extracting good properties of the SNLMS and the APSA. Moreover, the practical NESM-APSA is a good approximation of the ideal but impractical ONESM-APSA.

\section{Conclusions}

An improved affine projection sign algorithm against non-Gaussian impulsive noise is introduced. It is based on adding an error scalar matrix to the cost function subject to a constraint on the norm of the filter update. The result can be viewed as a generalization of the conventional APSA. Various nonlinear error transformation methods can be used in the new scheme with a little modification. An ideal nonlinear error scalar matrix is introduced based on the two component Gaussian mixture noise model, and we also discuss a practical one to approximate the optimal learning curve. Their computational complexities are both given. As shown in simulations, they obtain better 
performance than the NSA, the APSA, and the SNLMS. This is because proposed algorithms extract the good properties of the APSA, which employ past input vectors to improve the convergence rate for correlated input signals, and nonlinear error transformation methods, which reduce the influence of wrong information.

\section{Acknowledgements}

The research work was supported by The Natural Science Foundation of Chongqing under Grant No.cstc2012jjA40008

\section{References}

[1]Arikan, O., Enis A.C., and Erzin, E.: 'Adaptive filtering for non-Gaussian stable processes', IEEE Signal Process. Lett. 1994, 1, (11), p. 163-165

[2]Vega, L.R., Rey, H., Behest, J., and Tresses, S.: 'A new robust variable step-size NLMS algorithm’, IEEE Trans. Signal Process., 2008, 56, (5), p. 1879-1893

[3]L. R. Vega, H. G. Rey, J. Benesty, and S. Tressens, "A robust adaptive filtering algorithm against impulsive noise,” in Proc. ICASSP-2007, Honolulu, HI, Apr. 2007.

[4]L. R. Vega, H. G. Rey, J. Benesty, and S. Tressens, "A stochastic model for a new robust NLMS algorithm,” in Proc. EUSIPCO-2007, Poznan, Poland, Sep. 2007.

[5]Wu, L.F., He, H.S., and Qiu, X.J.: 'An active impulsive noise control algorithm with logarithmic transformation', IEEE Trans. Audio Speech Lang. Process., 2011, 19, (4), p. 1041-1044

[6] X. Sun, S. M. Kuo, and G. Meng, “Adaptive algorithm for active control of impulsive noise,” J. Sound Vibr, vol. 291, no. 1-2, p. 516-522, Mar. 2006.

[7]Song, I.S., Park, P.G., and Newcomb, R.W.: 'A normalized least mean squares algorithm with a step-size scalar against impulsive measurement noise', IEEE Trans. Circuits and Systems II: Express Briefs., 2013, 60, (7), p. 442-445

[8] M. T. Akhtar and W. Mitsuhashi, "Improving performance of FxLMS algorithm for active noise control of impulsive noise,” J. Sound Vibr, vol. 327, no. 3-5, p. 647-656, Nov. 2009.

[9]Shao, T., Zheng, Y.R., and Benesty, J.: 'An affine projection sign algorithm robust against impulsive interference’, IEEE Trans. Signal Process. Lett. 2010, 17, (4), p. 327-330

[10]H. C. Shin, A. H. Sayed, and W. J. Song, "Variable step-size NLMS and affine projection algorithms,” IEEE Signal Process. Lett, vol. 11, no. 2, p. 132-135, Feb. 2004.

[11]Ni, J.G., and Li, F.: 'Efficient implementation of the affine projection sign algorithm', IEEE Signal Process. Lett. 2012, 19, (1), p. 24-26

[12]J. Ni and F. Li, "Variable regularisation parameter sign sub band adaptive filter,” Electron. Lett. vol. 46, no. 24, p. 1605-1607, Nov. 2010. 